\title{
Die digitale Bibliothek erfordert digitale Fachkenntnisse
}

\author{
Die Ausbildung zum Fachinformatiker/Systemintegration
}

Wenn Einrichtungen ausbilden, so werden damit mindestens zwei Aspekte verfolgt: Jugendliche bekommen zum einen die Chance auf den Erwerb einer soliden Ausbildung, um ihnen einen guten Einstieg in das Berufsleben zu ermöglichen. Auf der anderen Seite erhalten die Ausbildungsbetriebe qualifizierte, gut eingelernte Fachkräfte, die notwendig sind, um den aktuellen Betrieb zu gewährleisten und gleichzeitig die weitere zukünftige Entwicklung zu gestalten. plexen Softwarelösungen und das Verständnis von IT-Zusammenhängen und IT-Standards. So fallen heute in Bibliotheken vielfältige IT-Aufgaben an. Exemplarisch sollen hier einige dieser Arbeitsbereiche aufgeführt werden: Geeignete Hardware im Nutzer-, Server- und Storagebereich muss beschafft, eingerichtet und verwaltet werden. Da Anwendungen in aller Regel netzwerkbasiert arbeiten, müssen Netzwerke konfiguriert, bedarfsorientiert ausgebaut und überwacht werden. Fachanwendungen müs-

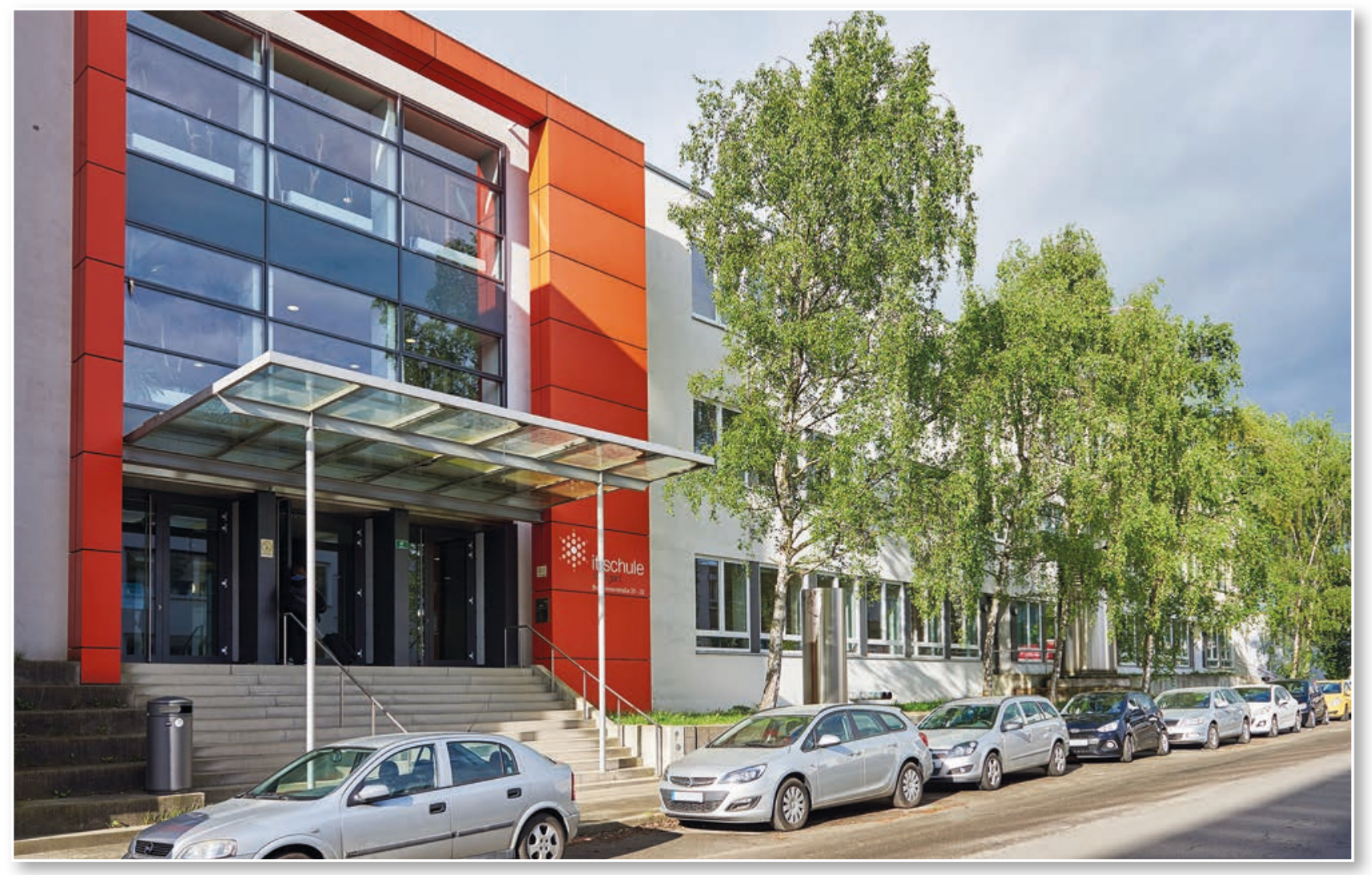

IT-Schule Stuttgart, Breitwiesenstraße (C) its

Durch den Einzug von digitalen Techniken und die Bereitstellung von Internetdiensten sind im Bibliotheksumfeld weitere Tätigkeitsprofile und neue Qualifikationsanforderungen hinzugekommen. Grundlage für die meisten Arbeitsprozesse und die Schaffung von bibliothekarischen Mehrwerten ist heute eine zuverlässige informationstechnische Infrastruktur, der sichere Umgang mit zum Teil kom- sen evaluiert, verteilt und aktualisiert werden. Für Bibliotheksbenutzer müssen IT-Arbeitsplätze und WLAN-Zugänge bereitgestellt werden. Daten müssen gesichert und ggf. einer Langzeitarchivierung unterzogen werden. Die Datenflüsse, der Datenaustausch und die Datenformate müssen abgesprochen, passend konvertiert, getestet und an neue Anforderungen angepasst werden. Der Aspekt der Datensicherheit muss berücksichtigt und Dokumen- 
tation erstellt und aktuell gehalten werden. Verschiedene Dienste müssen durch Programme und Skripte gekoppelt und Datenabläufe automatisiert werden. Eingeführte Dienste und Anwendungen müssen an neue Umgebungen und Sicherheitsanforderungen angepasst werden.

All diese Tätigkeiten stellen in immer größerem Maßstab originäres unmittelbares bibliothekarisches Handeln dar und können nicht einfach nur „nebenher" per Beauftragung erledigt werden. Dieses Handeln bedarf solider Fachkenntnis, stetiger Betreuung und entsprechend ausgebildeten Personals und muss sich auch in der Personalstruktur abbilden.

Die neuen Aufgabenfelder sollten sich auch in den Ausbildungsbemühungen der Bibliothek widerspiegeln. Die IHK bietet den Ausbildungsberuf Fachinformatiker in den beiden Ausprägungen „Systemintegration" und "Anwendungsentwicklung" an. Wie die obige beispielhafte Auflistung zeigt, besteht ein Großteil der bibliothekarischen IT-Aufgaben in der Integration, der Verknüpfung und Konfiguration von Systemen, so dass der Ausbildungsschwerpunkt "Systemintegration“ unsere Bedürfnisse sehr gut abdeckt.

Im Jahr 2004 wurde in der Württembergischen Landesbibliothek die Möglichkeit geschaffen, die Ausbildung für das Berufsbild "Fachinformatiker / Systemintegration " anbieten zu können. Diese duale Ausbildung dauert in der Regel drei Jahre und besteht aus einer allgemeinen, informationstechnischen Theorieausbildung, die im Rahmen des Berufschulbesuchs vermittelt wird, und aus dem Praxisteil im Ausbildungsbetrieb, in dem IT-Wissen konkret für die Einrichtung im bibliothekarischen Kontext zur Anwendung kommt. Neben der Vermittlung von technischen Grundlagen stehen in dieser Ausbildung auch Inhalte der Betriebsorganisation, Fragen der Arbeitsmethoden, Grundlagen zum Rechnungswesen und die Bedeutung des wirtschaftlichen Handelns im Fokus. Damit die Datenverarbeitung den bibliothekarischen Erfordernissen gerecht werden kann, wird im Rahmen der betrieblichen Ausbildung auch bibliothekarisches Grundwissen vermittelt. Da in der Württembergischen Landesbibliothek auch regelmäßig die FaMlAusbildung angeboten wird, konnten die "IT-ler" sich an internen FaMI-Schulungsangeboten und Rundgängen durch das Haus in den verschiedenen Abteilungen oftmals anhängen. Die Ausbildung wird mit einer zweiteiligen Abschlussprüfung beendet. In der Theorieprüfung müssen informationstechnische und kaufmännische Aufgaben schriftlich bearbeitet werden. Der praktische Prüfungsteil besteht in der kompletten Bearbeitung eines Prüfungsprojektes. Hierbei soll der Prüfling zeigen, dass er selbstständig eine Aufgabe in einem vorgegebenen Zeitfenster unter gegebenen wirtschaftlichen Rahmenbedingungen bearbeiten kann. Das Prüfungsthema wird dabei vom Ausbildungsbetrieb vorgeschlagen und der IHK zur Genehmigung vorgelegt. Im Abschluss an die Projektdurchführung muss der Prüfling das Projektergebnis der Prüfungskommission präsentieren, erläutern und verteidigen.

In der Württembergischen Landesbibliothek wurden in Rahmen der Prüfungsprojekte Themen aus den Bereichen Virtualisierung und Storagesysteme bearbeitet. All diese Themen konnten anschließend in der WLB nutzbringend eingeführt und produktiv zur Anwendung gebracht werden. Wer ausbildet, übernimmt Verantwortung, und Ausbilden macht auch Arbeit. Da die IT-Abteilung der WLB eher eine kleine Abteilung ist, konnten wir daher nicht mehr als einen Ausbildungsplatz pro Ausbildungsgang betreuen. Das ist sicherlich für den "Lehrling" eine etwas ungewöhnliche Situation, aber wir hatten den Eindruck, dass unsere Auszubildenden damit ganz gut zurechtkamen. Positiv ist auch zu erwähnen, dass die Mitarbeiterinnen und Mitarbeiter in der Bibliothek den Azubis sehr konstruktiv gegenüberstanden, ihr Wissen bereitwillig und geduldig teilten und sich viel Zeit genommen haben, um ihr Fachgebiet zu erläutern.

Ausbilden ist eine spannende Angelegenheit: Man hat es mit jungen Menschen zu tun und bekommt dadurch einen frischen, oftmals ganz neuen, unbefangenen Blick, auf die unterschiedlichsten Fragestellungen. Gerade im dynamischen informationstechnischen Umfeld machen wir immer wieder die Erfahrung, dass im Zuge der Ausbildung eine Wechselwirkung zwischen den Ausbildenden und Auszubildenden entsteht: Wir lernen gegenseitig voneinander. 